IDDF2018-ABS-0240 ROLE OF HUMAN MICROBIOTA IN SKIN DERMATITIS AND ECZEMA: A SYSTEMATIC REVIEW

${ }^{1}$ Learn-Han Lee*, 'Vengadesh Letchumanan, ${ }^{2}$ Tahir Mehmood Khan, ${ }^{3}$ Ming Long, ${ }^{4}$ KokGan Chan, ${ }^{5}$ Bey-Hing Goh, ${ }^{6}$ Nurul-Syakima Ab Mutalib. ${ }^{1}$ Novel Bacteria and Drug Discovery (NBDD) Research Group, School of Pharmacy, Monash University Malaysia, Malaysia; ${ }^{2} T$ The Institute of Pharmaceutical Sciences (IPS), University of Veterinary and Animal Sciences (UVAS), Pakistan; ${ }^{3}$ School of Pharmacy, KPJ Healthcare University College, Malaysia; ${ }^{4}$ Division of Genetics and Molecular Biology, Institute of Biological Sciences, Faculty of Science, University of Malaya, Malaysia; ${ }^{5}$ Biofunctional Molecule Exploratory (BMEX) Research Group, School of Pharmacy, Monash University Malaysia, Malaysia; ${ }^{6}$ UKM Medical Molecular Biology Institute (UMBI), UKM Medical Centre, University Kebangsaan Malaysia, Malaysia

\subsection{6/gutjnl-2018-IDDFabstracts.38}

Background Human gut microbiota - are normal flora living freely in the human gastrointestinal systems and this symbiotic bond begins at birth, and there are associations between human microbiota and skin allergens.

Methods This systematic review provides an insight into the role and condition of human microbiota that affects skin dermatitis and eczema. A thorough search was conducted using predefined terms in several electronic databases (PubMed, Medline, ScienceDirect), from database inception to March 2018. Studies must involve human microbiota, dermatitis, eczema, and full texts needed to be available. A total of 20 eligible articles were identified.

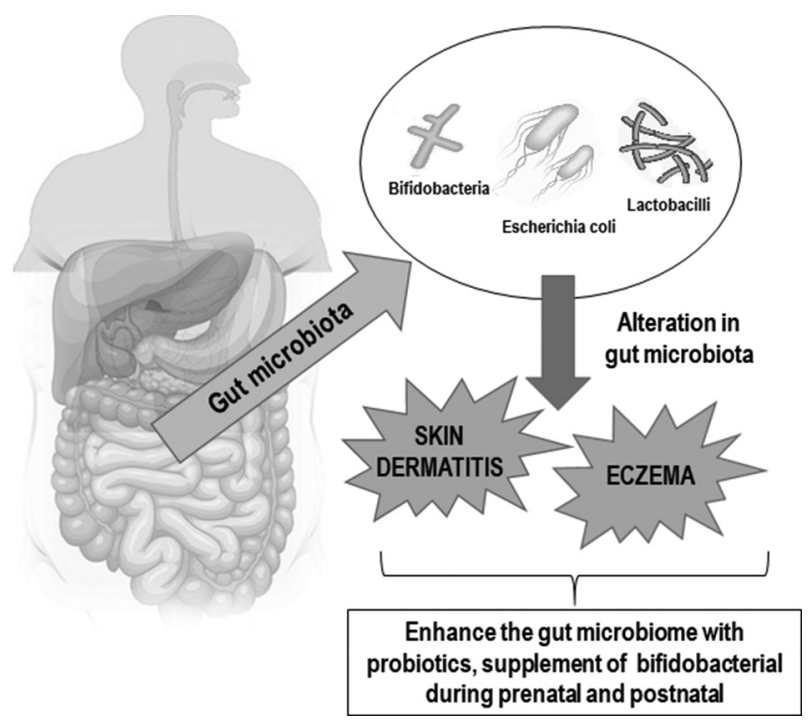

Abstract IDDF2018-ABS-0240 Figure 1

Results Based on the literature, several factors involving gut microbiota were identified that could affect skin dermatitis and eczema. Findings suggest that the dysbiosis of allergy in adults develops postnatally. At infancy stage, colonisation of the gut microbiota takes place and affects the immune development into the adolescence and adulthood. Hence, maternal milk represents a key factor to build ad modulate establishment of infant gut microbiota. Low level of $\operatorname{IgA}$ and $\operatorname{IgE}$ secretion in the gut system could reduce gut microbiota diversity. These antibody levels could be used to determine the establishment of a healthy symbiosis with gut microbiota.
Interactions between dysbiosis in Faecalibacterium prausnitzii and gut epithelial is noted as underlie cause for the progression of dermatitis. In addition, studies reported a reduction of the abundance of Bifidobacterium in the gut microbiota had been seen in infants with eczema. A variation of Bifidobacterium colonisation patterns in early life is said to be associated with later development of eczema or dermatitis. Figure 1 illustrates what happens when an alteration takes action to the normal microbiome flora such as Bifidobacterial, Escherichia coli and Lactobacilli. This leads to the development of dermatitis and eczema.

Conclusions In summary, probiotics can enhance and maintain a healthy gut microbiota. Studies also suggested prenatal and postnatal supplementation of bifidobacterial may be effective in the primary prevention of allergy diseases. These findings provide vital insight and knowledge that could enhance the potential for future microbial-based therapies to improve the clinical outcome of dermatitis and eczema.

\section{IDDF2018-ABS-0241 EPIGENETICS OF MUCINOUS COLORECTAL ADENOCARCINOMA THROUGH INTEGRATED TRANSCRIPTOME, METHYLOME AND MIRNOME ANALYSIS}

${ }^{1}$ Nurul Syakima Ab Mutalib*, ${ }^{1}$ Ryia Illani Mohd Yunos, ${ }^{1}$ Rasyidah Baharuddin, ${ }^{1}$ Noraidatulakma Abdullah, ${ }^{2}$ Luqman Mazlan, 'Rahman Jamal. 'UKM Medical Molecular Biology Institute, Universiti Kebangsaan Malaysia, Malaysia; ${ }^{2}$ Department of Surgery, Faculty of Medicine, Universiti Kebangsaan Malaysia, Malaysia

\subsection{6/gutjnl-2018-IDDFabstracts.39}

Background Mucinous cancer (MC) is a distinctive subtype of colorectal cancer (CRC) identified in 10\%-15\% of patients. It has long been associated with poor response to treatment, low survival rates, worse prognosis and present at more advanced stage than non-mucinous adenocarcinoma. Yet, the knowledge on the molecular mechanisms associated with this type of malignancy is lacking. This study aims to elucidate the transcriptome, methylome and miRnome landscape of MC for a better understanding of the disease and to find the potential target of therapy.

Methods We obtained the TCGA-generated methylation data based on Illumina Methylation 450 beadchip, level 3 microRNA expression and RNA sequencing dataset from Firebrowse. Gene expression data from 41 normal colons and methylation data from 45 normal colons were included for comparisons. Unpaired t-test with multiple testing corrections was performed using Bioconductor in $\mathrm{R}$ version 3.4.3.

Results Despite originating from the similar organ, $\mathrm{MC}$ and AC have distinct molecular profiles as shown by a high number of differentially methylated and expressed genes. We identified 2420 significant demethylated genes, and 3899 differentially expressed genes in MC. LY6G6D is the most downregulated gene while MUC2, the commonly discussed gene in MC, is the most upregulated. There were only four significant differentially expressed microRNAs with modest fold change, suggesting that DNA methylation is a more dominant epigenetic event in gene regulation of MC. EIF6 is the most significantly hypermethylated (25.6\% hypermethylation; $\mathrm{p}=4.18 \mathrm{E}-10)$ in $\mathrm{MC}$ versus $\mathrm{AC}$ and its expression was downregulated -0.57 fold $(\mathrm{p}=4.89 \mathrm{E}-07)$. On the other hand, $B A G 3$ is the most significantly hypomethylated $(22.1 \%$ 